\author{
Military Technical College \\ Kobry El-kobbah, \\ Cairo, Egypt
}

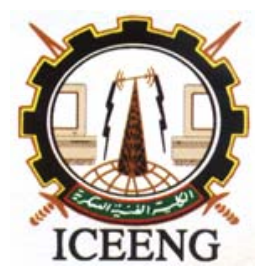

\author{
$5^{\text {th }}$ International Conference \\ on Electrical Engineering \\ ICEENG 2006
}

\title{
A Comparison between Optimal and Conventional Power System Stabilizers for a Single Machine to an Infinite Bus Power System
}

By
W. Sabry
A. Eliwa
H. A. Hassan
M. A. Talaat

Egyptian Armed Forces

\section{$\underline{\text { Abstract }}$}

The excitation control of synchronous generator in a single machine to an infinite bus (SMIB) power system is very important to improve the stability modes of power system. In addition to the usual or conventional excitation control techniques and speed governors, other devices proved its effectiveness to enhance better system stability like power system stabilizers (PSS) and static VAR compensators (SVC). In this paper, the PSS is used as an additional device to improve the SMIB power system stability. Two out of different control strategies were used; a conventional control technique (PI controller) and a linear optimal controller (LOC). A comparison between both types of PSSs was stated. The contribution of this paper is the design and application of an optimal-PSS and a PI-PSS to a synchronous generator connected to an infinite bus power system.

\section{$\underline{\text { Keywords }}$}

Single machine to an infinite bus, power system stabilizer, excitation control, PI controller, linear optimal controller

\section{Introduction}

The excitation control of synchronous generators is important to improve the stability of power system. For several decades, it has attracted researchers' attention. The literature review on excitation control is extensive and has different techniques [1].

To improve power system stability, supplementary excitation control design was used for low frequency oscillations. Power system stabilizers (PSSs) were 
already being investigated [2] and many papers had been published on this subject. Also, the coordinated application of PSSs in multi-machine power systems was presented [3].

The idea of supplementary excitation control is to apply a signal through the excitation system to increase the damping torque of the generator in a power system. At the application beginnings of such type of PSS, the proportionalintegral (PI) control technique was used [4].

Since the early seventies, the optimal control theory has been widely applied for the design of excitation control of synchronous generator and a method for selecting parameters of stabilizers in multi-machine power system was addressed [5].

The linear optimal controller (LOC) of electric power systems presented is derived from the minimization of the state variable deviations and control effort at the same time. The system state equations, or the state variable equations, must be sought first. Then, a performance index of the system is chosen. Finally, the state equations are appended to the performance index by a co-state variable vector to find the LOC. The co-state variable vector in modern control theory corresponds to the Lagrange multipliers in classic mechanics [5].

To a large extent, the above features correspond to the stability control problem in power systems. The contribution of this paper is the design and application of a LOC-PSSs to synchronous generator excitation controller connected to an infinite bus power system. Also, this article will compare the results deduced with the PI-PSS as known and widely used control type in power stations [6-7].

The paper is organized as follows: the principle of LOC is briefly introduced in section 2. The design procedure of the controller is described in section 3 . The simulation performance results of the controller are demonstrated and the variability of such a control strategy is established and further research is suggested in section 4 .

\section{Synthesis of the LOC}

Consider the linearized model of electric power system with respect to an initial steady state represented and including control by:

$$
\dot{X}=A X+B U
$$


Where: $X=\left(\mathrm{x}_{1} \mathrm{x}_{2} \ldots \mathrm{x}_{\mathrm{n}}\right)^{\mathrm{T}}$ is a state vector, $A$ is the system matrix, $B$ is the control matrix and $U$ is the control vector.

For the LOC design, a performance index of the quadratic form is usually chosen:

$$
J=\frac{1}{2} \int_{0}^{\infty}\left[X^{T} Q X+U^{T} R U\right] d t
$$

$Q$ is the weighting matrix of the state variable deviations and $R$ that of the control effort. LOC is derived from the minimization of the performance index. The Hamilton generalized-energy function:

$$
H \equiv \frac{1}{2}\left[X^{T} Q X+U^{T} R U\right]+P^{T}[A X+B U]
$$

$P$ is the co-state vector that corresponds to Lagrange multipliers. To find the LOC, the following condition must be satisfied:

$$
\partial H / \partial U=0
$$

Carrying out the differentiation:

$$
R U+B^{T} P=0
$$

And the control rule becomes:

$U=-R^{-1} B^{T} P$

For conciseness, let:

$$
S=B R^{-1} B^{T}
$$

From Equations (5, 6 and 7):

$$
B U=-B R^{-1} B^{T} P=-S P
$$

Partial differentiation of $H$ in Equation (3) with respect to $X$ and $P$, respectively, gives:

$$
\dot{X}=\partial H / \partial P=A X+B U=A X-S P
$$


And

$$
\dot{P}=-\partial H / \partial X=-\left(Q X+A^{T} P\right)
$$

Equations (9 and 10) may be written together to give system equation as:

$$
\left[\begin{array}{l}
X \\
P
\end{array}\right]^{\bullet}=\left[\begin{array}{cc}
A & -S \\
-Q & -A^{T}
\end{array}\right]\left[\begin{array}{l}
X \\
P
\end{array}\right]
$$

To find the LOC, it is necessary to find the solution of the co-state variable vector $P$. $P$ can be related to $X$ linearly by: $P=K X$ where $K$ is the Riccati matrix. Substituting in Equation (11):

$$
K A+A^{T} K-K S K+Q=0
$$

Using iterative methods to solve Equation (12) for $K$.

\section{Power systems with LOC}

In this paper, we consider the power system is a single generating unit connected to an infinite bus, which is shown in Figure (1) [6].

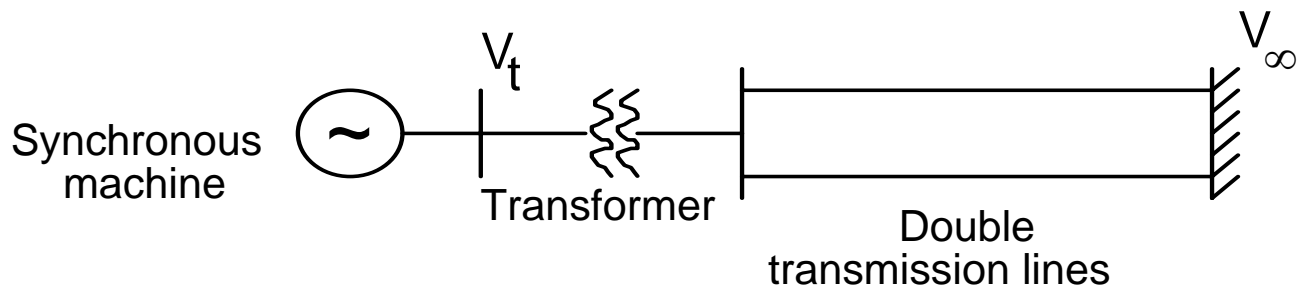

Figure (1): A single generator infinite system

The model of the system can be written as in Equation (5). The state vector is given by:

$$
X=\left[\begin{array}{ll}
\Delta \delta & \Delta \omega
\end{array}\right]^{T}, A=\left[\begin{array}{cc}
0 & 377 \\
-K_{1} / M & D / M
\end{array}\right] \text { and } B=\left[\begin{array}{ll}
0 & 1 / M
\end{array}\right]^{T}
$$

Once the system has been established, the next step is to construct LOC design procedure and getting its parameters. For $M=10, D=0, f=60 \mathrm{~Hz}$ and $K_{1}=0.5$, the eigen-values are: $\lambda= \pm j 4.342$ elec. Rad./s, which is critically stable system. For the given data: 


$$
A=\left[\begin{array}{cc}
0 & 377 \\
-0.05 & 0
\end{array}\right] \text { and } B=\left[\begin{array}{ll}
0 & 0.1
\end{array}\right]^{T}
$$

For the LOC design let:

$$
Q=\left[\begin{array}{cc}
0.25 & 0 \\
0 & 1
\end{array}\right] \text { and } R=1
$$

From Equation (7), the $S$ matrix will be: $S=\left[\begin{array}{cc}0 & 0 \\ 0 & 0.01\end{array}\right]$ then solve Equation (12) to get $K$. It will be: $K=\left[\begin{array}{cc}0 & 2.071 \\ 2.071 & 395.3\end{array}\right]$. The new system eigen-values becomes: $\lambda$ $=-1.9765 \pm j 4.769$, which is a stable system.

\section{$\underline{\text { 4. Simulation results }}$}

In the simulation study, we consider that the power system shown in Figure (1) with a large sudden fault at $100 \mathrm{~ms}$ and the fault was removed at $250 \mathrm{~ms}$. The response of power angle $\delta(\mathrm{t})$ and generator terminal voltage $\mathrm{V}_{\mathrm{t}}(\mathrm{t})$ are shown in Figures (2) and (3), which are controlled by the LOC (solid line) and general PI controller (dash line).

From the simulation results given above, we see that using the LOC proposed in this paper can maintain transient stability and achieve voltage regulation and has better dynamic performance using the general PI controller.

\section{$\underline{\text { 5. Conclusion }}$}

In this paper, transient stabilization and voltage regulation of power system with a LOC have been discussed. The LOC scheme has been given. The variable structure excitation control proposed in this paper can transiently stabilize a class of power systems with a large sudden fault and can achieve voltage regulation. Simulation results show that the variable structure excitation controller possesses a good dynamic performance. 
Proceedings of the $5^{\text {th }}$ ICEENG Conference, 16-18 May, 2006

EP $-3-6$

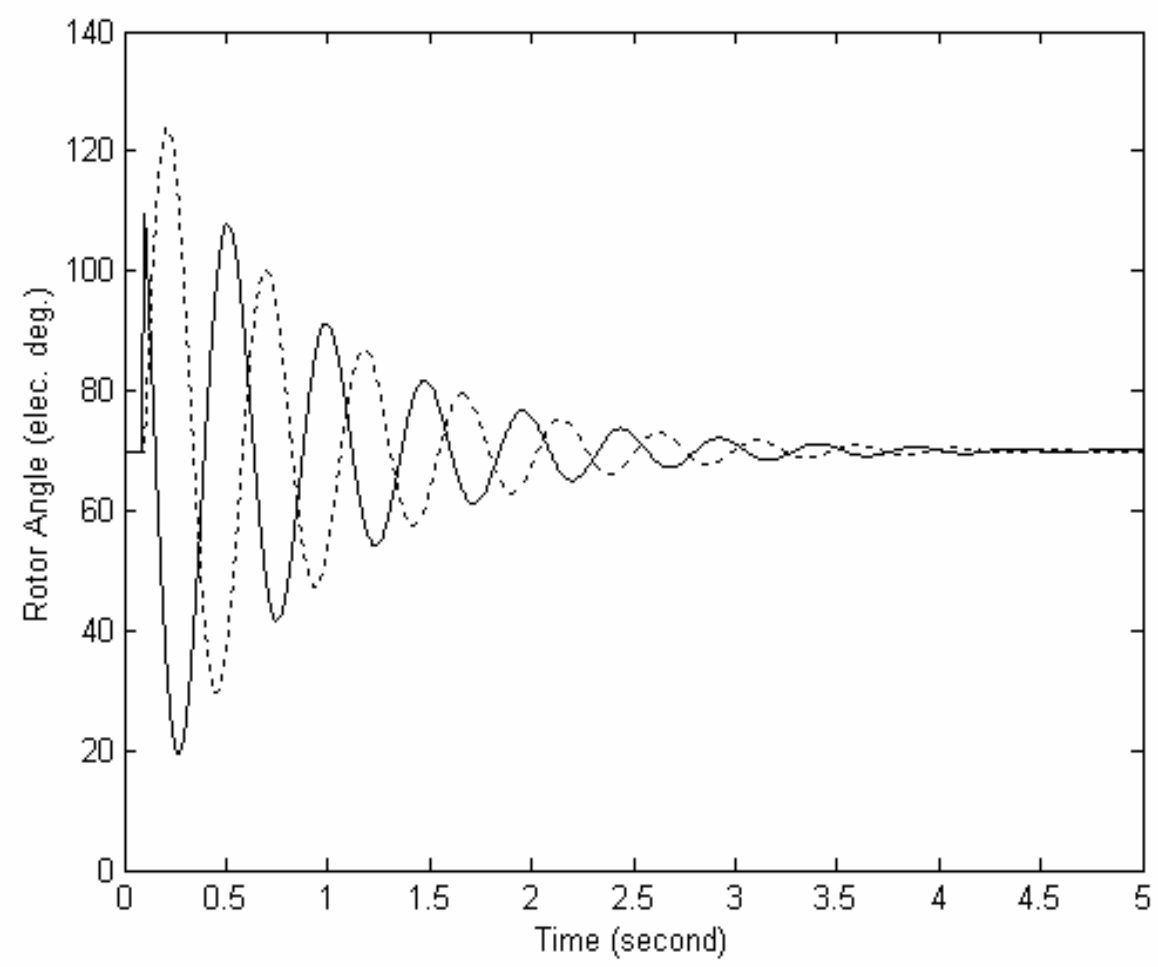

Figure (2): The rotor angle response

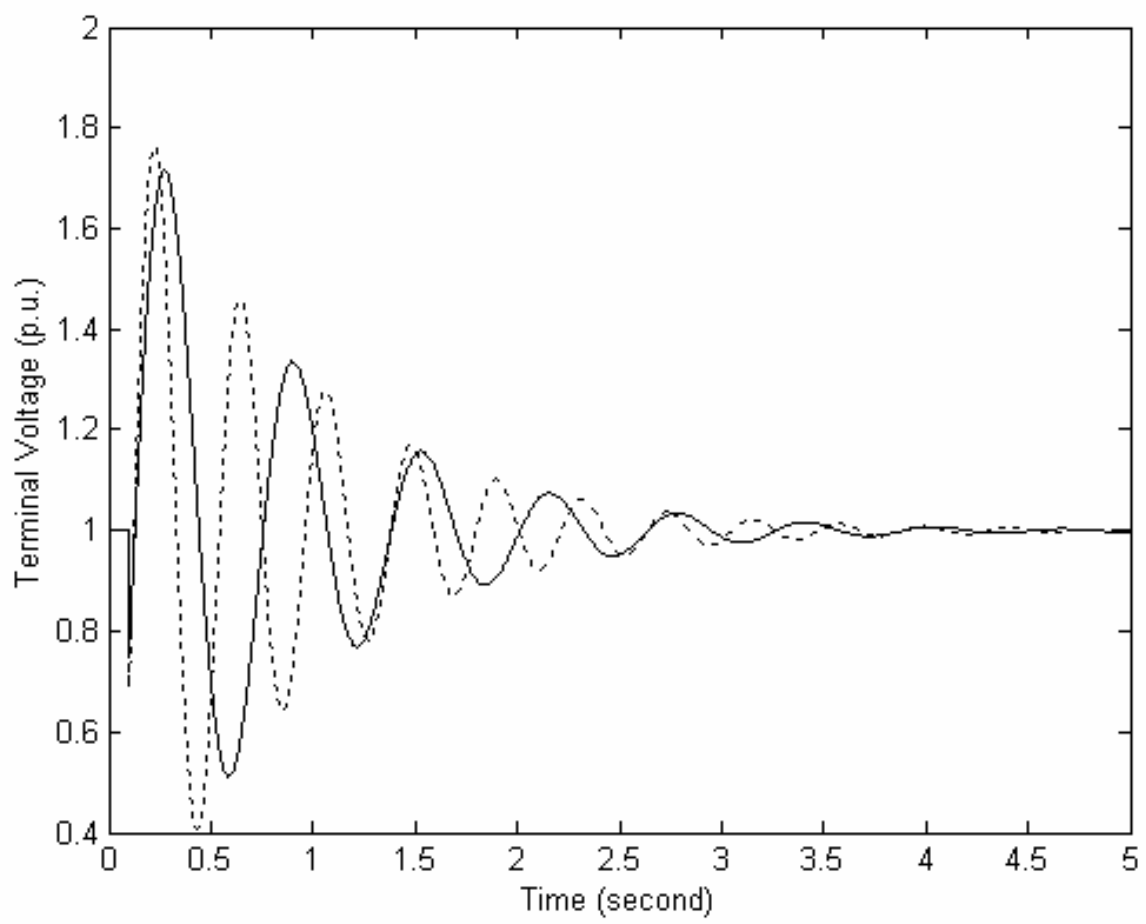

Figure (3): The voltage response 


\section{$\underline{\text { References }}$}

[1] DeMello, P. F. and C. Concordia, Concepts of synchronous machine stability as affected by excitation system control, IEEE Trans. On Power Apparatus and Systems, PAS-88, No. 2, 1969.

[2] Moussa, H. A. and Yu, Y. N., Dynamic interaction of multimachine power system and excitation control, IEEE Trans. On Power Apparatus and Systems, PAS-94, No. 4, 1975.

[3] DeMello, P. F. et al., Coordinated application of stabilizers in multimachine power systems, IEEE Trans. On Power Apparatus and Systems, PAS-99, No. 3, 1980.

[4] Yu, Y. N. and Siggers, C., Stabilization and optimal control signals for a power system, IEEE Trans. On Power Apparatus and Systems, PAS-90, No. 4, 1971.

[5] Yu, Y. N., Electric power system dynamics, Academic Press, 1983.

[6] Lu, Q. and Y. Sun, Nonlinear control of power systems, Chinese Academic Press, 1993.

[7] Utikin, I. V., Sliding modes in control and optimization, Springer-Verlag, Berlin, Heideberg, 1992. 